\title{
Unraveling the embryonic fate map through the mechanical signature of cells and their trajectories
}

David Pastor-Escuredo ( $\nabla$ david@lifedlab.org )

CNRS \& UPM https://orcid.org/0000-0002-2662-8686

\section{Benoit Lombardot}

MPI-CBG

\section{Thierry Savy}

CNRS

\section{Adeline Adeline Boyreau}

CNRS

\section{Rene Doursat}

CNRS

Jose Maria Goicolea

Universidad Politénica de Madrid

\section{Andres Santos}

Universidad Politecnica de Madrid https://orcid.org/0000-0001-7423-9135

\section{Paul Bourgine}

\author{
CNRS
}

\section{Juan Carlos del Alamo}

UCSD

Maria Jesus Ledesma-Carbayo ( $\square$ mledesma@upm.es )

UPM

\section{Nadine Peyrieras ( $\nabla$ nadine.peyrieras@cnrs.fr)}

CNRS

\section{Article}

Keywords: embryo morphogenesis, mechanical cues, digital cell lineages, tissue deformation, biomechanical signature

Posted Date: March 16th, 2021

DOl: https://doi.org/10.21203/rs.3.rs-333921/v1

License: (9) This work is licensed under a Creative Commons Attribution 4.0 International License. 



\title{
Unraveling the embryonic fate map through the mechanical signature of of cells and their trajectories
}

\author{
David Pastor-Escuredo a,b,", Benoît Lombardot ${ }^{\mathrm{c}}$, Thierry Savy ${ }^{\mathrm{a}, \mathrm{c}}$, Adeline Boyreau ${ }^{\mathrm{a}}$, René \\ Doursat $^{\mathrm{c}}$, Jose M. Goicolea ${ }^{\mathrm{d}}$, Andrés Santos ${ }^{\mathrm{b}}$, Paul Bourgine ${ }^{\mathrm{a}, \mathrm{c}}$, Juan C. del Álamo ${ }^{\mathrm{e}, \mathrm{f}, 1}$, María J. \\ Ledesma- Carbayo $^{\mathrm{b}, 1,{ }^{*}}$ and Nadine Peyriéras ${ }^{\mathrm{a}, \mathrm{c}, 1,{ }^{*}}$
}

\begin{abstract}
Affiliations:
aUSR3695/FRE2039 BioEmergences, CNRS, Paris-Saclay University, Gif-sur-Yvette, France. biomedical Image Technologies, ETSIT, Technical University Madrid, 28040, Spain \& Biomedical Research Center in Bioengineering, Biomaterials and Nanomedicine (CIBER-BBN), Spain. 'UPS3611 Complex Systems Institute Paris Ile-de-France (ISC-PIF), CNRS, Paris, France ${ }^{\mathrm{d} C}$ Computational Mechanics Group, Technical University Madrid, 28040, Spain.

eMechanical and Aerospace Engineering Department, University of California San Diego, La Jolla, CA 92093, United States.

fInstitute for Engineering in Medicine, University of California San Diego, La Jolla, CA 92093, United States.

*Corresponding authors: nadine.peyrieras@cnrs.fr, mledesma@die.upm.es, david@lifedlab.org

${ }^{1}$ These authors equally contributed
\end{abstract}

\begin{abstract}
Digital cell lineages reconstructed from 3D+time imaging data of the developing zebrafish embryo are used to uncover mechanical cues and their role in morphogenesis. A continuous approximation of cell displacements obtained from cell lineages is used to assess tissue deformation during gastrulation. At this stage, embryonic tissues display multi-scale compressible fluid-like properties. The deformation rate at the mesoscopic level of the cell's immediate surroundings appears noisy, in both space and time. The patterns identified by clustering the cells, according to the cumulative deformation rate along their trajectory throughout gastrulation, lead to a robust, ordered and coherent biomechanical map. The timing and amplitude of the biomechanical deformations provide a measurement of the phenotypic variability in small cohorts of specimens. We show that the biomechanical map matches the embryonic fate map of the zebrafish presumptive forebrain, in both wild type and Nodal pathway mutants $\left(z o e p^{t z 57 / t z 57}\right)$, where it reveals the biomechanical defects that lead to cyclopia.. The comparison of biomechanical patterns and the expression pattern of a transgenic reporter for the
\end{abstract}


transcription factor goosecoid ( $g s c$ ), supports the hypothesis that embryonic cells acquire, at an early developmental stage, a biomechanical signature that contributes to defining their fate.

\section{Introduction}

Embryonic morphogenesis depends on the mechanical properties of the cells that make up tissues, in conjunction with the generation and transduction of forces that control their interactions ${ }^{1,2}$. Mechanical stimuli affect all levels of biological organization, including gene expression and the signaling activities that underlie both cell proliferation and cell death ${ }^{3,4}$. Cell stiffness, contractility, protrusive activity and adhesive properties define the mechanical properties of tissues. The robustness of embryonic development depends on the coordination of biochemical and biomechanical processes in space and time ${ }^{5-8}$. Live microscopy $3 \mathrm{D}+$ time imaging permitted the automated reconstruction of digital cell lineages and cell shapes ${ }^{9-16}$, which opened the way to a more quantitative and formal approach of cell dynamics and tissue deformation ${ }^{17,18}$. The quantitative analysis of cell displacements and shape changes has been used to characterize the tissue deformation rates in 2 dimensions $(2 D)$ in Drosophila or zebrafish embryos, and how certain mutations affect these parameters ${ }^{18-20}$. The construction of models that describe tissue properties and cell contacts succeeded to evaluate mechanical stresses in $2 D^{21-25}$ and more recently in $3 D^{26}$. The challenge ${ }^{27-28}$ that remains is to tackle processes in $4 D$ (3D over time), and then correlate biomechanical patterns with other spatiotemporal features, such as changes in gene expression and determination of cell fate ${ }^{29-31}$. Because its embryos develop rapidly and are transparent, the zebrafish is the vertebrate model of choice to acquire live imaging data and investigate the spatial and temporal dynamics of tissue deformation that underlie vertebrate gastrulation ${ }^{32-34}$. 
Kinematic descriptors computed from digital cell lineages ${ }^{35,36}$ show the rates with which tissues deform during zebrafish gastrulation. These deformations include compression or expansion, rotation and distortion in $3 D$ on the mesoscopic scale of a cell's immediate surroundings. The deformation rates at the level of the cell's neighborhood fluctuate locally both in space and time. We find that these fluctuations are compensated through a cumulative process that operates during gastrulation along cell trajectories. We arrive at a set of mechanical descriptors of the cell's neighborhood that constitute its biomechanical profile. We applied machine learning to these biomechanical profiles and built a mechanical signature for every single cell along its trajectory ${ }^{37,38}$. The ability to visualize cell populations in space and time, clustered according to the similarity of their mechanical signature, showed ordered and coherent patterns. Differences observed between wild type $(w t)$ and mutant embryos $\left(z o e p^{t z 57 / t z 57}\right.$, deficient for the transduction of the Nodal pathway ${ }^{39}$ ) showed that in mutants, altered deformation patterns anticipate the cyclopic phenotype. These findings prompted a comparison with morphological landmarks and gene expression patterns. We find that the embryonic fate map can be inferred from cellular mechanical history without any knowledge of their identity or fate.

\section{Results}

\section{Deformation rates provide landmarks for zebrafish gastrulation}

We constructed a biomechanical map of the developing organism with cells' biomechanical profiles calculated from strain descriptors obtained from a tensorial analysis (overview in Figure 1). We quantified mechanical strains at the (mesoscopic) scale of the cell and its neighborhood from cell lineage trees reconstructed from $4 D$ imaging data of zebrafish embryos developing from $6 \mathrm{hfp}$ to $14 \mathrm{hpf}^{36}$ at $26^{\circ} \mathrm{C}$ (Figure 1). Our methodology was applied to six wild-type embryos (wt1-wt5, wt6)(Supplementary Movie 1,2), one of them carrying a GFP reporter to 
monitor the expression of goosecoid $(\mathrm{gsc})$ transcription factor, and five $z o e p^{\mathrm{tz} 57 / 257}$ mutants (oep1- oep5, Supplementary Movie 3) with deficiency in the transduction of Nodal signals that leads to cyclopia (Supplementary Table 1). Embryos were imaged from the animal pole throughout gastrulation (Figure 1A, Supplementary Fig. 1) to observe the development of their presumptive head region and forebrain (temporal resolution $2.5 \mathrm{~min}$./frame; voxel size : $1.4 \mu \mathrm{m}^{3}$ (Supplementary Movie 1, Supplementary Table 1)).

Mechanical strains were calculated from a regularized vector flow field $v_{T R}$ (Figure $1 \mathrm{~B}$ and Figure 2 first column), obtained through temporal averaging of cell displacements by a Gaussian kernel filter $(T=10 \mathrm{~min})$ and their spatial regularization. The optimal regularization term resulted in a characteristic spatial scale of interpolation of $R=20 \mu \mathrm{m}$. Depending on developmental stage, this scale covers one or two rows of neighboring cells as cell size diminishes throughout gastrulation. Artifacts can originate from cell tracking errors ${ }^{25}$ (Supplementary Fig. 2), which are in part removed by this operation. Tracking errors were further corrected automatically to ensure continuity of cell trajectories (Figure 1C). This is necessary to analyse the evolution over time of the kinematic descriptors.

The tensorial analysis was accomplished by first calculating the Incremental Deformation Gradient (IDG) tensor field ${ }^{40,41}$ to build a time-evolving Eulerian description (Figure 1D). The Eulerian description of deformation includes expansion and compression rates $(P<0$ and $P>0$ respectively), distortion (shear) rate $\left(Q_{d}\right)$ and rotation discriminant $(D)$ (Figure 1D and Supplementary Table 2, 4). The distortion rate descriptor $\left(Q_{d}\right)$ derived from the deviatoric tensor ${ }^{3}$, allowed quantitation of the amount of mesoscopic shape changes as a consequence of intercalation of cells and/or changes in cell shape. 
The different descriptors were displayed as spatiotemporal maps with our visualization interface Mov-IT ${ }^{36}$. This display showed characteristic patterns of mechanical activity in the zebrafish anterior region between 6 and 14 hours post fertilization (hpf) at $26^{\circ} \mathrm{C}$ (Supplementary Movie 49). The Eulerian descriptor maps provided a new class of spatiotemporal landmarks, characteristic of morphogenetic transitions.

For wild-type embryos, at the onset of gastrulation (6 hpf), planar expansion of the blastoderm over the yolk cell showed that tissue shaping still involved epiboly movements only, with cells moving away from a source point in the velocity field $v_{T R}$, located at the animal pole (Figure 2, Supplementary Fig. 3, Supplementary Movie 4-9). The zoep $p^{\mathrm{tz57/257}}$ mutant embryos showed a similar behavior (Supplementary Fig. 4, Supplementary Movie 10). As gastrulation proceeded (7-8 hpf), tissue compression at the dorsal side of the embryo was the predominant mechanical activity. Consequently, a mechanical boundary emerged at the anterior border of the neural plate, as revealed by the velocity $v_{T R}$ and the compression and expansion rate $P$. The $z o e p^{\mathrm{tz5} 7 / 257}$ mutant embryos still showed similar behavior but with this mechanical boundary shifted posteriorly. By mid-gastrulation of wild type embryos (8-9 hpf), the distortion rate $\left(Q_{d}\right)$ indicated shear along the midline throughout the tissues, likely the result from the relative movements of hypoblast (internal cell layers) and epiblast (external cell layers) (Figure 2, Supplementary Fig. 3, 5, Supplementary Movie 11-15). This behavior was not seen in the mutant embryos (Supplementary Movie 16,17), in agreement with their known defect in hypoblast formation. The defective hypoblast did not have the proper speed to slide under the epiblast (Supplementary Fig. 4). Massive mesoscopic distortion $\left(Q_{d}\right)$ and drop of compression at the time of tail bud closure (10-11 hpf) was consistent with fast convergence of cells toward the midline and with their intercalation (Supplementary Fig. 3, 5). The mutant embryos showed an increase in tissue 
compression at the level of their shifted dorso-ventral mechanical border (Supplementary Fig. 4). By 9 to $12 \mathrm{hpf}$ in $w t$ embryos, increasing rotation rates $(D)$ on both sides of the midline marked the end of gastrulation and the onset of neurulation, with clockwise rotation contributing to tissue convergence toward the midline and antero-posterior axis elongation.

By $12 \mathrm{hpf}$, the disappearance of large-scale biomechanical deformations indicated another regime of tissue dynamics (Figure 2). zoep $p^{\mathrm{tz5} 7 / 257}$ mutant embryos did not display the characteristic vortices observed by the end of gastrulation in $w t$ embryos. Together with the marked posterior shift of the dorso-ventral mechanical border, this feature suggested that the midline in the mutant failed to play its role of repellant and only kept its role of attractor.

The mechanical patterns unveiled by the Eulerian descriptors (Figure 2, Supplementary Movie 4$9,17)$ in wild-type embryos showed heterogeneities in space and time. While the velocity $\left(v_{T R}\right)$ field was fairly symmetric, at least from 6 to $10 \mathrm{hpf}$, the pattern of the topology descriptor $(\tau)$, showed bilateral heterogeneity (Supplementary Fig. 3, Supplementary Movie 4-9, 17). This descriptor, $\tau$, combines compression $(P)$ and rotation $(D)$ measurements and integrates their fluctuations. Its persistence in time was blurred by its instantaneous fluctuations. While constrained in the tissue flow of gastrulation, neighboring cells experienced (on a 10 min time scale) variable levels of strain. Mutant embryos also showed heterogeneities within a single specimen and even more variability across the mutant specimens analyzed (Supplementary Fig. 4, Supplementary Movie 10,17). The loss of symmetries seemed to produce greater instability in the embryos.

The robustness of biomechanical cues results from the cumulative rates of deformation along cell trajectories 
We hypothesized that cumulating the mechanical constraints that cells experience over discrete time intervals might allow the emergence of more robust and persistent biomechanical patterns (Figure 3). We relied on a Lagrangian trajectory-based representation of the descriptors to assess the cumulative deformation rates along cell trajectories. This representation defined Lagrangian Biomechanical Profiles (LBPs), which will be further classified and averaged in Canonical Lagrangian Biomechanical Profiles CLBPs (Figure 1E-G). Computing series of IDG tensors along the trajectories integrated from the vector flow field $v_{T R}$ (Supplementary Movie 18) provided a sequence of Finite-Time Deformation Gradient (FTDG) tensor fields. Cumulative mechanical cues along the trajectories from an initial temporal reference $t_{i n i}$ were then expressed by the invariants of the FTDG tensors (Supplementary Table 3, 4). The latter included volume changes $(\Delta V)$, rotation angle $(\Delta \alpha)$ and tissue distortion or reshaping $\left(\Delta \gamma_{1}\right.$ and $\left.\Delta \gamma_{2}\right)$ for the amount of distortion and its geometrical configuration respectively (Figure 3, Supplementary Fig. 6, Supplementary Movie 19, 20). The cumulative LBPs led to spatially more homogeneous territories than the Eulerian descriptors (Supplementary Movie 18-20) suggesting, that throughout gastrulation, neighboring cells experienced similar cumulative mechanical deformations (Figure 3, Supplementary Fig. 7).

\section{Interindividual comparison based on the Lagrangian Biomechanical Profiles of selected cell populations}

To further investigate the homogeneity of cumulative versus instantaneous mesoscopic mechanical cues in cell populations, and the robustness of the patterns in different wild-type zebrafish embryos, we investigated different ways of decreasing the dimensionality of the data. This was achieved by defining cell populations either a priori according to embryological knowledge or without any a priori hypothesis using machine learning methods. The mean and 
variance of instantaneous LBPs for similar cell domains encompassing part of the dorsal epiblast and hypoblast selected manually in five different embryos ${ }^{42}$ (tailbud selection, Supplementary

Fig. 8, Supplementary Movie 21, 22) confirmed temporal markers for the progression of gastrulation in both tissues (Figure 4A, Figure 2). Interindividual comparison confirmed the robustness of biomechanical features but also indicated timing differences, which we interpreted as a consequence of variability in experimental conditions (e.g. temperature). The onset of epiblast compression was taken as a landmark to temporally align the different datasets at an initial state $\left(t_{i n i}\right)$ between 7-8 hpf) and calculate the cumulative profiles (Figure 4B). As expected from results in Figure 3, cumulative LBPs produced more homogeneous and robust patterns than the instantaneous ones, reinforcing the hypothesis of spatiotemporal compensation of local fluctuations.

\section{Canonical Lagrangian profiles (CLBPs) characterize cell populations mechanical history}

We further investigated the spatiotemporal coherence of biomechanical patterns by systematically categorizing the cumulative LBPs for a selection of cells selected that remain in the field of view from 8 to 14 hpf (shield-stage selection, Supplementary Fig. 9). The selected cell population is located at the animal pole of the early gastrula and was expected to encompass the presumptive forebrain and the underlying prechordal plate ${ }^{29}$. Similarities amongst the LBPs were estimated based on their dynamic range and temporal evolution. We calculated the LBP distance distribution using a cosine metric and unsupervised classification to find groups with minimal variance. This classification created categories of LBPs and the corresponding canonical Lagrangian Biomechanical Profiles (CLBPs) for each of the descriptors (Figure 5A). Three CLBPs sufficed to characterize the diversity of mesoscopic deformation histories within the selected cell populations (Figure 5A, Supplementary Fig. 10-12). For each of the descriptors, 
one of the canonical profiles appeared to segregate the hypoblast from the epiblast, indicating its phenotypic homogeneity (Supplementary Fig. 12). Conversely, the epiblast population appeared heterogeneous, as two different canonical profiles were required to characterize its cells' mechanical history (Supplementary Fig. 12).

\section{The biomechanical map of $o e p^{t 57 / t z 57}$ mutants predicts their cyclopic phenotype}

The temporal and spatial coherence and robustness of the cumulative descriptor patterns suggested that unsupervised machine learning could reveal morphogenetic domains with distinct mechanical histories. The cell trajectories were hierarchically classified according to their signature in terms of CLBPs (Supplementary Fig. 13). This uncovered four main populations ordered in space and time. These populations were coherent and formed bilaterally symmetrical domains that can now be compared with the state-of-the-art brain fate map ${ }^{29-31}$ (Figure 5B, Supplementary Fig. 14-16, Supplementary Movie 23-25). We used our interactive visualization tool Mov-IT to compare the CLBP based categories to morphological compartments, as identified by fluorescent staining of the cell membrane. The fluorescently stained domain was confined at the posterior midline by $8 \mathrm{hpf}$. We interpret this as having captured the prechordal plate and the ventral forebrain, i.e. presumptive hypothalamus. This region undergoes an early increase in distortion and rotation, followed by a rigid anterior displacement over the yolk. The similar mechanical histories of these two fields highlight the role of the prechordal plate in the formation of the hypothalamus and in the separation of bilateral eyes. The three other populations (Fig. 5; yellow, pink and red) matched the eye field and probably the ventral telencephalon. The eye field appeared to contain three domains with different mechanical histories: i) a most ventral domain (yellow) that underwent the steepest temporal increase in rotation by the end of gastrulation, ii) a ventral medial domain (pink) that underwent a late 
distortion and low-to-intermediate compression, and iii) a ventral lateral (red) subjected during gastrulation and early neurulation to the highest increase in compression (Supplementary Fig. 15, 16, Supplementary Movie 23-25). Altogether, we propose that the unsupervised classification of canonical LBPs identifies morphogenetic fields and anticipates their regionalization based on a characterization of the cumulative mechanical cues along cell trajectories (Supplementary Fig. $16,17)$.

We hypothesized that morphogenetic defects such as cyclopia should lead to major biomechanical perturbation at an early stage of development. This was shown by the biomechanical map of zoep $p^{t z 57 / t z 57}$ mutants. zoep $p^{t z 57 / t z 57}$ mutants defective in the transduction of Nodal signals lack endoderm and endomesodermal cells of the prechordal plate ${ }^{43}$ (Supplementary Movie 26). The calculation of CLBPBs showed a reduced distortion activity in mutants compared to wild type embryos (Figure 5A bottom row, Supplementary Fig. 17). As a consequence, the biomechanical map constructed for zoep $p^{t z 57 / t z 57}$ embryos gathered 3 fields instead of 4 in wild type (Figure 5B). The main perturbation at an early step of gastrulation in zoep ${ }^{t z 57 / z 57}$ embryos is the absence of the green domain at the midline, characterized by its high shear activity. This defect leads to a cascade of abnormalities that include delayed convergence of cells towards the midline and antero-posterior extension of the body axis. We interpret from the biomechanical map, that the midline acts as an attractor-repeller structure in wild type embryos and that it retains only its attractor activity in zoep ${ }^{t z 57 / t z 57}$ mutants. This leads to the absence of the vortices normally observed in the cell flow in wild type embryos (Supplementary Movie 9,10 ) and to the absence of separation of the red biomechanical domain at the midline (Fig. 5B, red domain) consistent with a unique eye field that leads to cyclopia. We hypothesized 
that the green biomechanical domain should match with the prechordal plate. It was assessed in gsc:egfp transgenic embryos with labeled prechordal plate ${ }^{44}$.

\section{Insights from the comparison between gene expression and biomechanical maps}

We constructed the biomechanical map of a gsc:egfp transgenic embryo (Figure 6, Supplementary Movie 27). The level of expression of the fluorescent reporter along cell trajectories was estimated (Methods). Three domains were identified with different levels of expression (high in green, medium in cyan and low in blue) (Figure 6A, Supplementary Fig. 18). In each group, artifacts in the intensity of the reporter expression due to depth and bleaching were compensated according to the intensity of nuclear staining that is expected to be homogeneous in space and constant in time (Figure 6B, 6C). The gsc reporter expression domains overlaps the green biomechanical domain. This supports the hypothesis that the biomechanical impact of the prechordal plate in tissue morphogenesis is essential to the formation of the forebrain (Figure 6D, 6E).

\section{Concluding remarks}

Digital reconstruction of cell lineage trees, based on 3D+time imaging of a developing organism is a new approach to study the cell and tissue dynamics that underlie morphogenesis ${ }^{45}$. The quantitative aspects of such datasets enable the construction of models with predictive value. The exploitation of these massive quantitative data sets for maximum effect requires the development of new interdisciplinary approaches State-of-the-art computational workflows were designed to process time-lapse imaging data sets and achieve cell tracking in space and time. Nonetheless these approaches have their limitations and do not produce error-free lineage trees ${ }^{35,36}$, especially 
in the case of vertebrate organisms with high cell density and thick tissues. A typical $2 \%$ error rate of false links per time step requires manual corrections to retrieve the cell's complete clonal history. We show that this somewhat noisy data set readily ends itself to kinematic analysis, based on an approximation of the tissue flow ${ }^{46}$. Gastrulating tissues in zebrafish display a fluidlike behavior, as suggested also for other vertebrate species (e.g. chicken ${ }^{47}$ ). A constitutive equation that would capture the relationship between spatially and temporally heterogeneous stresses/strains within embryonic tissues has yet to be formulated. To do so will require stress measurements in the whole organism, a major challenge. Our 3D framework for automated kinematic analysis of tissue deformation can uncover a possible role for mechanical cues in morphogenesis. Although the forces that drive zebrafish gastrulation remain to be determined, our 3D kinematic analysis shows that displacement of the hypoblast relative to the epiblast at the dorsal midline generates bilateral jet-vortices that shape the anterior brain, including bilateral eyes. Our 3D kinematic patterns and their comparison with gene expression maps provide a new set of tools to study what establishes morphogenetic fields and the resulting presumptive organs. More generally, 3D instantaneous and cumulative tissue deformation patterns, as validated here for zebrafish gastrulation, can provide insights in morphogenesis for unknown species. The method is well suited for large-scale collective cell displacements, imaged and reconstructed with state-of-the-art tools.

Biomechanical fate maps integrate diversity, robustness and spatio-temporal organization of mechanical patterns. Our results establish that mechanical interactions at the cell scale and at the tissue scale play a key role in driving developmental outcomes.

\section{Methods}

\section{D+time imaging data of a cohort of zebrafish embryos}


Wild-type or $o e p^{t z 57 / t z 57}$ mutant Danio rerio (zebrafish) embryos were stained as described by ${ }^{48}$, RNA injection at the one-cell stage with 100pg H2B-mCherry and 100pg eGFP-HRAS mRNA (Supplementary Fig. 1) prepared from PCS2+ constructs $^{48,49}$. gsc:egfp embryos were counterstained with $\mathrm{H} 2 \mathrm{~B}$-mCherry. Embryos raised at $28.5^{\circ} \mathrm{C}$ for the next 3 hours were dechorionated and mounted in a 3-cm Petri dish filled with embryo medium. To position the embryo, the Petri dish had a glass coverslip bottom, sealing a hole of $0.5 \mathrm{~mm}$ at the dish center, holding a Teflon tore (ALPHAnov) with a hole of $780 \mu \mathrm{m}$. The embryo was maintained and properly oriented by infiltrating around it $0.5 \%$ low-melting-point agarose (Sigma) in embryo medium ${ }^{50}$. Temperature in the Petri dish slightly differed for the 6 wildtype specimens (wt 1 : about $25^{\circ} \mathrm{C}$ estimated, $w t 2: 26^{\circ} \mathrm{C} w t 3: 26^{\circ} \mathrm{C} w t 4: 28.6^{\circ} \mathrm{C} w t 5: 24.7^{\circ} \mathrm{C} w t 6: 26^{\circ} \mathrm{C}$ ) given by a temperature probe in the Petri dish (OKOLAB). After the imaging procedure, the embryo morphology was checked under the dissecting binocular and the animal was raised for at least 24 hours to assess morphological defects and survival. The different datasets encompassed the same developmental period (4-6 hpf to 14-16 hpf). All the specimens were imaged from the animal pole and the imaged volume encompassed the forebrain with some differences depending on the animal positioning in its mold. Variability in the development speed reflects temperature differences as well as intrinsic variability of embryonic development. Imaging was performed as described ${ }^{36}$ with 2-photon laser scanning ${ }^{51}$ on Leica SP5 upright microscopes and high numerical aperture 20x water dipping lens objectives. Image acquisition parameters are summarized in Supplementary Table 1.

\section{Digital cell lineages}

The $3 \mathrm{D}+$ time datasets featured a constant time step $\Delta t$ of approximately 2.5 minutes, defining a discrete time scale for the cohort and a voxel size between 1.2 and $1.4 \mu m^{3}$ (Supplementary 
Table 1). Given this time interval in comparison with the temporal lengthscale of the gastrulation, we assume instantaneous observation, therefore $\Delta t \sim \delta t$. Digital cell lineages (Figure 1, Supplementary Fig. 1) were obtained through the BioEmergences automated image processing workflow ${ }^{36}$. Cell positions were given by the approximate nucleus centers after applying the nuclei segmentation module of the workflow. The cell lineage data including cell positions at each time step, linkage from one time step to the other and linkage between mother and daughter cells at the time of cell division was computed by the cell tracking module and presented in a comma-separated-values (.csv) table format (cell identifier and position, mother identifier called as such whether the cell divides or not). As reported in Faure et al. ${ }^{36}$, after manual corrections made through the tool Mov-IT, the BioEmergences tracking method yielded an error rate of approximately $2 \%$ representing the percentage of false or missing links between two consecutive time steps $[t, t+\delta t]$.

\section{Flow field approximation of cell lineage}

We used the cell lineage to provide the complete structured spatiotemporal information about cell trajectories, including cell divisions. Specifically, we proposed a generalized data record for each cell nucleus within the lineage as follows:

$\{\text { cellid, motherid, } x, y, z, t\}_{i}$

where $i$ indexes the detected cells (a new $i$ is created at each mitosis and allocated to one of the daughter cells), cellid is the corresponding unique identifier of the local node representing cell $i$ at the $(x, y, z, t)$ spatio-temporal nucleus position in the lineage tree, and motherid is the identifier of the linked nucleus position at the previous time step (mother cell). The set of detected nuclei forms a discrete spatiotemporal map

Gaussian filtering of the velocity field with temporal information 
Given a temporal resolution of the time-lapse data of $\delta t \sim 2.5$ minutes, singular cell displacements produced by divisions or tracking errors occurred at a frequency close to the sampling's Nyquist frequency and were thus assumed to generate high-frequency noise. In order to filter out this noise, we performed a temporal smoothing of the displacements along the cell lineage with a Gaussian kernel $N(0, T)$, where $T$ is a scale in the order of minutes. By testing several parameters, we set $T$ $=10$ min to modify the samples of the original velocity field at $x_{i}$ and generate a smoothed velocity field $v_{T}\left(x_{i}\right)$ with the same temporal resolution and spatial distribution. In addition, displacements over the threshold MaxMov $=9 \mu \mathrm{m} / \delta t$ were removed as outliers. Overall, this filtering helped remove and smooth out vectors resulting from segmentation and tracking errors.

$$
\begin{gathered}
v_{T}\left(x_{i}(t)\right)=\frac{1}{\alpha_{i, R}} \sum_{t^{\prime} \in \Pi} \quad w_{i, T}\left(t^{\prime}\right) v_{T}\left(x_{i}\left(t+t^{\prime}\right)\right) \\
w_{i, T}=e^{-\frac{t^{2}}{2 T^{2}}} \text { if } i \text { is well tracked } \\
w_{i, T}=0 \text { if not } \\
\Pi=\{t+k \delta t \mid k \text { integer } k \delta t \in[-2 T, 2 T]\} \\
\alpha_{i, T}(t)=\sum_{t^{\prime} \in \Pi} w_{i, T}\left(t^{\prime}\right)
\end{gathered}
$$

\section{Regularization of the velocity field with spatial information}

The vector field $v_{T}$ was further filtered around the position of each nucleus $x_{i}$ through a spatial Gaussian kernel $N(0, R)$ obtaining smoothed versions of the velocity field $v_{T R}\left(x_{i}\right)$ with the same temporal resolution and the same spatial distribution of samples. Through a regularization process based on the Gaussian filtering characterized by the parameter $R$ and a second-order structure function derived from the field, we tested the differentiability of the new vector field $v_{T R}\left(x_{i}\right)$ and adjusted the parameter $R$ to minimally modify the samples velocity field.

The spatial Gaussian filtering was defined as follows: 


$$
\begin{gathered}
v_{T R}\left(x_{i}(t)\right)=\frac{1}{\beta_{i, R}} \sum_{j \in N_{R}(i)} w_{i, R}\left(x_{j}\right) v_{T}\left(x_{i}(t)\right) \\
w_{i, R}=e^{-\frac{t^{2}}{2 R^{2}}} \\
\beta_{i, T}(t)=\sum_{t^{\prime} \in N_{R}} w_{i, R}\left(x_{j}\right)
\end{gathered}
$$

where $N_{R}(i)=\left\{x_{j}\right\}$ is the set of neighbors interpolated, $w_{i, R}\left(x_{j}\right)$ is the weight of each neighbor according to the $N(0, R)$ distribution and $\beta_{i, R}$ the sum of all the weights. In order to preserve boundaries within the displacement field, a binary function $S\left(x_{j}, x_{i}\right)$ was used to discard outlier displacements:

$$
N_{R}(i)=\left\{j \mid\left\|x_{j}-x_{i}\right\|<2 R, S\left(x_{j}, x_{i}\right)=1\right\}
$$

where $S\left(x_{j}, x_{i}\right)$ comprises three thresholds based on data observation: the maximum angle of deviation against the reference $(\pi / 2)$, a minimum speed $(0.2 \mu \mathrm{m} / \mathrm{min})$ and a maximum ratio of speed against the reference $\left(3 v_{T}\left(x_{i}\right)\right)$.

To calibrate the spatial filtering $N(0, R)$ and impose local differentiability of the velocity field around each sample $x_{i}$, we used a second-order structure function. As the initial velocity field, the vector field $v_{T R}\left(x_{i}\right)$ represents a discrete and inhomogeneously sampled field. The differentiability of a random field is directly related to the differentiability of its covariance $R$ at the origin. Experimentally, from a practical point of view, it is easier to accurately measure the structure function than the covariance, which is why we use the structure function. The two functions are related as $R(l)=R(0)-S 2(l) / 2$ so it does not matter from a fundamental point of view which one is used. The classic result is that $v$ is continuous if $S 2(l)->0$ with $l->0$ and that $v$ is differentiable if $S 2(l) \sim l^{2}$ as generally described in 52. Structure functions are often used in noisy flow fields (e.g. turbulence) to determine whether the velocity field is smooth $(u(x+$ 
$l)-u(x) \sim l)$ as opposed to Holder continuous $\left((u(x+l)-u(x) \sim l)^{\alpha}\right.$ with $\left.\alpha<1\right)$ within a certain lengthscale range $(l)$. Thus, to fit our inhomogeneous and sparse field, we quantify the differentiability of this field using a second-order structure function built in terms of the average velocity differences within $n$ concentric rings around each $x_{i}$. Local differentiability is therefore observed when the structure function follows a power law with exponent $\geq 2$. The structure function is defined as:

$$
S 2_{i}[n]=\left\{\left\langle\left\|v_{T R}\left(x_{k}\right)-v_{T R}\left(x_{i}\right)\right\|^{2}\right\rangle\right\}_{\varphi_{n, i}},
$$

where $n \in[1,10]$ and $\varphi_{n, i}$ denotes that we calculated the discretized function $S 2_{i}[n]$ around each position $x_{i}$ in concentric rings with the same radius $d_{l}$, i.e.

$$
\varphi_{n, i}=\left\{k \mid(n-1) d_{l}<\left\|x_{k}-x_{i}\right\|<n d_{l}\right\}
$$

We determined the regularized vector field $v_{T R}$ and the kernel width $R$ considering the local differentiability of all field samples. To this end, we computed a time-dependent ensemble average of the $S 2_{i}[n]$ function for each ring $S 2(n, t)=\left\langle S 2_{i}[n]\right\rangle_{\varphi_{n}^{\prime}}$, where $\varphi_{n}^{\prime}$ is the subset of all the rings derived from $\varphi_{n, i}$ after removal of outlier rings. We labeled a ring as an outlier when the function $S\left(x_{j}, x_{i}\right)$ described above for the cells within the ring was negative for the majority of them, indicating largely divergent information in the ring that, if included in the regularization term, could over smooth the field globally. Testing several parameters, an optimal kernel width was found, $R=20 \mu \mathrm{m}$, that ensured differentiability while minimally worsening the spatial resolution of the displacement field (Supplementary Fig. 2).

\section{Instantaneous deformation descriptors}

The differentiability of the vector flow field $v_{T R}$ allowed us to apply principles of continuum mechanics to quantify cell motion and tissue deformation. We calculated the Incremental Deformation Gradient (IDG) tensor field $f\left(x_{i}\right)$ by a numerical method (least squares error 
minimization) considering the displacements of the neighboring cells within the volume defined by $2 R$. The IDG tensor for the cell $i$ at position $x_{i}^{t}$ and time $t$ defines a mapping from the material (i.e., Lagrangian) vector difference $d x_{i}^{t}$ onto the vector $d x_{i}^{t+\Delta t}$ :

$$
\begin{gathered}
d x_{i}^{t+\Delta t}=f d x_{i}^{t} \\
x_{i}^{t+\Delta t}=x_{i}^{t}+v_{T R}\left(x_{i}^{t}\right) \delta t
\end{gathered}
$$

We obtained flow topology descriptors as Galilean invariants derived from the tensor Incremental Gradient of Displacements $h=f-I$ (Supplementary Table 2, Figure 1). These invariants are tensor metrics that are independent of the reference frame orientation and velocity. They are therefore suitable for visualizing and comparing complex $3 D$ flows. The first, second and third principal invariants in $3 D$ (referred here as invariants) are interpreted as follows. The first invariant of $h(P$ as described in 41) quantifies the compression/expansion rate at the mesoscopic scale, corresponding to an overall decrease/increase of cell size change in the vicinity of each nucleus. The second invariant ( $Q$ as described in 41) gives information about deformation, not producing volume changes at the mesoscopic scale, associated with both irrotational and vortical motions. We identified the deformation produced by rotation the discriminant of the deformation tensor $(D)$, which is positive in regions of mesoscopic rotation. Finally, we designed a topology index descriptor $\tau$ that takes four different values representing the combinations of the signs of the descriptors $P$ and $D$ (Figure 2, Supplementary Fig. 3-5): expansion-rotation (green label), expansion-no rotation (blue label), compression-rotation (yellow label) and compression-no rotation (red label).

To further characterize the strain rates, we calculated the symmetric part of the tensor $h$ that stands for the irrotational, incremental strain tensor $\varepsilon$ and its principal components. This symmetric tensor generally provides information about shears and changes in volume through its second invariant $Q_{s}$. For tissues that may change volume and in order to distinguish between reconfigurations of 
the tissues (cell intercalation and cell shape changes) and volume changes (cell size), we calculated the deviatoric tensor $d$ that subtracts volume changes from the strain rate. The eigenvectors $\left\{d_{1}, d_{2}, d_{3}\right\}$ and second invariant $Q_{d}$ of this tensor provided information on the tissue distortion associated to collective cell intercalation and cell shape changes (Supplementary Table 2, Figure 2, Supplementary Fig. 3-5).

The description of the vertical motion can be complemented with the angular velocity, computed from the skew-symmetric tensor $\Omega$ derived from $h$. The infinitesimal rotation angle $\delta \alpha$ was derived from .

\section{Building Lagrangian Biomechanical Profiles}

We defined a Lagrangian representation of the flow field by approximating the reconstructed cell trajectories by the flow path lines of the regularized vector flow field. Lagrangian analysis of noncompressible $(P=0)$ two-dimensional flows have been successfully applied to discover Lagrangian Coherent Structures in fluid transport ${ }^{53-54}$. Here, we proposed Lagrangian metrics based on the computation of finite-time deformation tensors to unfold the biomechanical history along the lineage. Because of cell divisions and incomplete cell trajectories, the reconstructed cell lineage had to be regularized to build a continuous flow description. We interpolated the cell trajectories with the information of the flow field displacements to generate complete trajectories given an interval of time $\left[t_{n}, t_{m}\right]$ (Supplementary Movie 17). Thus, we generated a bijective spatiotemporal map $\operatorname{Traj}_{n}^{m}=\left\{\operatorname{traj}_{j}\right\}$ suitable to express the dynamics in terms of trajectories (Lagrangian) $v_{T R}\left(\operatorname{traj}_{j}\right)$ instead of spatial points (Eulerian).

We built Lagrangian Biomechanical Profiles (LBPs) independent of spatial coordinates using the trajectory flow field. LBPs expressed the instantaneous and cumulative biomechanical activity along each trajectory (Figure 3, Supplementary Fig. 6, 7, Supplementary Movie 18, 19). The 
cumulative activity was computed for each trajectory by setting a temporal reference $t_{i n i}$ and by incrementally enlarging the interval of analysis to generate a sequence of FTDG tensors $F^{t_{i n i}}\left(\operatorname{traj}_{j}\right)$ (varying along the time interval of the trajectory) and their corresponding descriptor fields. The computation of the FTDG tensor and its invariants (Supplementary Table 3, 4) for each interval $\left[t_{\text {ini }}, t\right]$ is described below.

\section{Computation of FTDG tensors and descriptors}

The IDG tensor field was expressed in Lagrangian terms using the trajectory field $f\left(\operatorname{traj} j_{j}\right)$ or $f_{j}^{t}$. We composed the tensors along each trajectory and the corresponding time interval with the chain rule (forward-projection matrix operation) to generate a Finite Time Deformation Gradient (FTDG):

$$
F_{j}^{\left[t_{i n i}, t\right]}\left\{\operatorname{traj}_{j}\right\}=f_{j}^{t-1} \ldots f_{j}^{t_{i n i}+1} f_{j}^{t_{i n i}}
$$

The third invariant of the tensor ( $\Delta V$ as described in 40) characterized the volume change during the time interval. The finite rotation (tensor $R$ ) was segregated from elongation (tensor $U$ ) through a polar decomposition $F=R U^{42}$. The rotation was described with the angle of rotation $\Delta \alpha$ and the axis of rotation (Euler's theorem as described in 40):

$$
\Delta \alpha=\left(\frac{\operatorname{tr}(R)-1}{2}\right)
$$

The strains were obtained with the right Cauchy-Green tensor $C=F^{T} F$ and the left Cauchy-Green tensor $B=F F^{T}$ and their principal components. The invariants of these tensors integrated volume changes and shear strains. Therefore, we calculated the isochoric deformation tensor and the corresponding isochoric Cauchy-Green tensors $(\tilde{C}$ and $\tilde{B})$ to identify the distortion along the trajectories from the volumetric changes:

$$
\tilde{F}=J^{-\frac{1}{3}} F
$$


The first and second $\tilde{C}$ and $\tilde{B}$ ( $\Delta \gamma_{1}$ and $\Delta \gamma_{2}$ as described in 37$)$ represented the tissue shape changes along the time intervals. The descriptor $\Delta \gamma_{1}$ evolved linearly with the amount of distortion whereas the descriptor $\Delta \gamma_{2}$ added second-order terms when the distortion had more than one dimension. Thus together, the $\Delta \gamma_{1}$ and $\Delta \gamma_{2}$ descriptors characterized the amount and geometry of tissue shape changes (Supplementary Table 3, Figure 3, Supplementary Fig. 6, 7). Aliases have been proposed for finite time descriptors as shown in Supplementary Table 3.

\section{Visualization of descriptor maps and manual selection of cell domains.}

The BioEmergences custom visualization tool Mov-IT ${ }^{36}$ was used to explore the $3 D+$ time descriptor maps. The maps for the Eulerian descriptors were computed by generating a color map for the IDG tensor values at each nuclear center $x_{i}$. For the cumulative LBPs, color maps were built and visualized with the Mov-IT software by assigning values to the closest nuclear center $x_{i}$. Mov-IT was also used to manually select cell domains and propagate the selections along the cell tracking, in order to perform a statistical analysis of the corresponding LBPs. Two different types of cell populations were selected. Expert embryologists selected cell populations at 10-11 hpf within the hypoblast and epiblast layers that were approximately similar in position and cell number between the five specimens of the cohort. These populations were backtracked to identify the corresponding progenitors at the onset of gastrulation (tailbud selection Figure 4, Supplementary Fig. 8, Supplementary Movie 21). We also selected in embryo wt1 by the onset of gastrulation the largest possible population of cells kept into the imaged volume throughout the

whole imaging sequence (shield selection, Figure 5, Supplementary Fig. 9, Supplementary Movie $22,23)$. This selection was used to categorize the different types of profiles with unsupervised classification.

\section{Categorization of Lagrangian Biomechanical Profiles}


A trajectory field defined from the shield cell selection (Supplementary Fig. 9) was further characterized by identifying subdomains with similar LBPs $l_{j}=l_{\text {traj }_{j}}(t)$ (descriptor along the trajectories). The subdomains were identified by generating a distance $d_{L B P}$ distribution between the LBPs $\left(l_{j}, l_{k}\right)$ of pairs of cells for each descriptor with a cosine metric, selected because it properly weighted both the magnitude of the descriptor and its deviations along time:

$$
d_{L B P}=d_{c o s}\left(l_{j}, l_{k}\right)=1-\frac{l_{j} l_{k^{\prime}}}{\sqrt{\left(l_{j} l_{j}\right)\left(l_{k} l_{k^{\prime}}\right)}}
$$

We applied unsupervised $k$-means clustering to classify the trajectories minimizing the variance of the distance distribution, so that trajectories with similar profiles according to the metric were classified together. The behavior of each cluster was defined using the mean of the trajectory profiles along time, which is considered suitable because the variance was minimized. Several values of the number of clusters $k$ were tested (Supplementary Fig. 10-12), finding that 3 clusters provided a suitable representation of Canonical Lagrangian Biomechanical Profiles (CLBPs) (Figure 5, Supplementary Fig. 10-12).

\section{Identifying morphogenetic domains from their mechanical signature}

We generated a mechanical signature $\zeta_{j}(\{C L B P\})$ for each trajectory as a binary feature vector based on the corresponding set of CLBPs (Figure 5, Supplementary Fig. 13). Trajectories were then compared using the Euclidean distance between their mechanical signatures and classified into four representative domains using hierarchical clustering ${ }^{55}$.

The obtained classification was used to label the nuclei at the onset of gastrulation $\left(t_{i n i}\right)$, generating Lagrangian Biomechanical Maps (Figure 5, Supplementary Fig. 14-16). The spatiotemporal evolution of the mechanical domains was visualized with Mov-IT by propagating the 
corresponding labels along the cell trajectories (Figure 5, Supplementary Fig. 14-16, Supplementary Movie 22-24).

\section{Identifying morphogenetic domains from their gsc signature}

Analogously, goosecoid ( $g s c$ ) profiles were computed from vectors sampling the level of gsc reporter expression ${ }^{41}$ channel along the trajectories (Figure 6). Each vector was computed by computed the average value of a spatial region around each trajectory point. Thus, the genetic expression was expressed in a Lagrangian representation to be compared with the mechanical profiles. A clustering module with the same configuration used for CLBPs returned the main gsc profiles. Figure 6 showed the spatio-temporal distribution of the clustered gsc domains in the embryo in comparison with the mechanical domains. Supplementary Fig. 18 shows the average of the Lagrangian descriptors normalized between 0 and 1 over time.

\section{Lagrangian comparison of embryo specimens based on their LBPs}

A comparison between specimens was made using the CLBPs of one embryo as the reference specimen (wtl). A time-varying similarity score was defined for each trajectory in the compared embryo $\beta_{L B P}^{r e f}(t)$. The score $\beta_{L B P}^{r e f}(t)$ represented the similarity of the CLBPs of the compared embryo with the reference CLBPs at each time point along the interval $\left[t_{\text {ini }}, t_{f i n}\right]$. The similarity score was here defined and computed as the distance of each descriptor CLBP $j$ to the corresponding CLBPs of the reference. Then, the smallest distance resulting from each CLBP $\mathrm{i}_{\mathrm{i}}$ was taken as the score.

$$
\beta_{L B P}^{r e f}(j, t)=\left\{d\left(C L B P_{j}(t), C L B P_{i}^{r e f}(t)\right)\right\}
$$

An aggregated similarity score $\beta_{\text {ave }}^{r e f}(t)$ was computed for each embryo as the average of the distance of all CLBPs per time step for each descriptor. Thus, the similarity score of each compared embryo to the reference was presented as a time evolving vector that conveys the information 
about the timing and temporal distribution of the variability of mechanical activity based on the reference. We used the CLBPs of the reference wild-type embryo (wtI) to compare two different wild-type embryos and two mutant embryos (Supplementary Fig. 17).

\section{Code availability}

Movies, raw images and cell lineage data are available on the BioEmergences website: http://bioemergences.eu/kinematics/ login: kinematics password: AGBYeL4y

All code is available upon request or already available at the BioEmergeces workflow ${ }^{36}$.

\section{Competing interests}

The authors declare no competing financial interests.

\section{Contributions}

DP developed the framework, designed visualization and analysis, interpreted the results and cowrote the manuscript. BL designed and developed the foundation of the study. TS developed the visualization and co-wrote the manuscript. $\mathrm{AB}$ carried out biological experimentation and imaging. RD supervised the final version of the Methods. JG supervised the physics part. AS cofunded and co-coordinated the project. PB co-funded and co-coordinated the project and supervised the foundation of the study. JCA co-funded and co-coordinated the project, supervised the physics part and co-wrote the manuscript. MJL co-funded and co-coordinated the project, supervised the design of the analysis framework, co-wrote the manuscript. NP co-funded and co-coordinated the project, supervised data acquisition and analysis, led the biological interpretation, co-wrote the manuscript.

\section{Acknowledgments}

We thank the BioEmergences laboratory, especially Louise Duloquin and Dimitri Fabrèges for their collaboration and support. This work was supported in part by TEC2015-66978-R, RTI2018098682-B-I00 by the Spanish ministry of Science, Innovation and Universities, co-funded by the European Union ERDF (European Regional Development Fund) and European Social funds for A.S. and M.L.C., by grants Project\# 1R01HD092216-01A1, NSF CBET - 1055697 and NIH R01 GM084227 for J-C.A. and by grants EC NEST "Measuring the Impossible" 28892, ZF-Health EC project HEALTH-F4-2010-242048, ANR BioSys Morphoscale, France BioImaging infrastructure ANR-10-INBS-04, ANR-11-EQPX-029 and InterDIM 2011 Région Paris Ile-de-France for N.P. and P.B.

\section{References}

1. Heisenberg C. P., Bellaïche Y. (2013) Forces in Tissue Morphogenesis and Patterning. Cell 153, 948-962. 
2. Lecuit T., Lenne P., Munro E. (2011) Force generation, transmission, and integration during cell and tissue morphogenesis. Annu. Rev. Cell Dev. Biol. 27, 157-184.

3. Bosveld F. et al. (2012) Mechanical control of morphogenesis by Fat/Dachsous/Four-jointed planar cell polarity pathway. Science 336, 724-727.

4. Bosveld, F. et al. (2016). Epithelial tricellular junctions act as interphase cell shape sensors to orient mitosis. Nature 530, 495.

5. Blanchard G., Adams R. J. (2011) Measuring the multi-scale integration of mechanical forces during morphogenesis. Curr. Opin. Genet. Dev. 21, 653-663.

6. Davidson L., von Dassow M., Zhou J. (2009) Multi-scale mechanics from molecules to morphogenesis. Int. J. Biochem. Cell Biol. 41, 2147-2162.

7. Keller R. Physical biology returns to morphogenesis (2012) Science 338, 201-203.

8. Gilmour, D., Rembold, M., \& Leptin, M. (2017). From morphogen to morphogenesis and back. Nature, 541(7637), 311.

9. Olivier N. et al. (2010) Cell Lineage Reconstruction of Early Zebrafish Embryos Using LabelFree Nonlinear Microscopy. Science 329, 967-971.

10. Supatto W., Truong T. V., Débarre D., Beaurepaire E. (2011) Advances in multiphoton microscopy for imaging embryos. Curr. Opin. Genet. Dev. 21, 538-548.

11. Keller P. J. (2013) Imaging Morphogenesis: Technological Advances and Biological Insights. Science 340, 1234168-1234168.

12. Gao L., Shao L., Chen B., Betzig E. (2014) 3D live fluorescence imaging of cellular dynamics using Bessel beam plane illumination microscopy. Nature protocols 9, 1083-1101. 
13. Steigmaier J. et al. (2016) Real-Time Three-Dimensional Cell Segmentation in Large-Scale Microscopy Data of Developing Embryos. Developmental Cell. 36, 225-240.

14. Wolff, C., Tinevez, J. Y., Pietzsch, T., Stamataki, E., Harich, B., Guignard, L., ... \& Pavlopoulos, A. (2018). Multi-view light-sheet imaging and tracking with the MaMuT software reveals the cell lineage of a direct developing arthropod limb. Elife, 7, e34410.

15. Liu, T. L., Upadhyayula, S., Milkie, D. E., Singh, V., Wang, K., Swinburne, I. A., ... \& Kohrman, A. Q. (2018). Observing the cell in its native state: Imaging subcellular dynamics in multicellular organisms. Science, 360(6386), eaaq1392.

16. Ovečka, M., von Wangenheim, D., Tomančák, P., Šamajová, O., Komis, G., \& Šamaj, J. (2018). Multiscale imaging of plant development by light-sheet fluorescence microscopy. Nature plants, $4(9), 639-650$.

17. McMahon A., Supatto W., Fraser S. E., Stathopoulos A. (2008) Dynamic analyses of Drosophila gastrulation provides insights into collective cell migration. Science 322, 1546-1550.

18. Behrndt M. et al. (2012) Forces Driving Epithelial Spreading in Zebrafish Gastrulation. Science $338,257-260$.

19. Blanchard G. B. et al. (2009) Tissue tectonics: morphogenetic strain rates, cell shape change and intercalation. Nature Methods 6, 458-464.

20. Tetley, R. J. et al. (2016). Unipolar distributions of junctional Myosin II identify cell stripe boundaries that drive cell intercalation throughout Drosophila axis extension. eLife, 5, e12094.

21. Graner F., Dollet B., Raufaste C., Marmottant P. (2008) Discrete rearranging disordered patterns, part I: Robust statistical tools in two or three dimensions. The European Physical Journal E 25, 349-369. 
22. Brodland G. W. et al. (2010) Video force microscopy reveals the mechanics of ventral furrow invagination in Drosophila. Proceedings of the National Academy of Sciences 107, 22111-22116.

23. Chiou K. K., Hufnagel L., Shraiman B. I. (2012) Mechanical Stress Inference for Two Dimensional Cell Arrays. PLoS Comput Biology 8, e1002512.

24. Ishihara S., Sugimura K. (2012) Bayesian inference of force dynamics during morphogenesis. $J$. Theoretical Biology 313, 201-211.

25. Tlili S. et al. (2015) Colloquium: Mechanical formalisms for tissue dynamics. The European Physical Journal E 38, 1-31.

26. Veldhuis, J. H., Ehsandar, A., Maître, J. L., Hiiragi, T., Cox, S., \& Brodland, G. W. (2017). Inferring cellular forces from image stacks. Philosophical Transactions of the Royal Society B: Biological Sciences, 372(1720), 20160261.

27. Polacheck, W. J., \& Chen, C. S. (2016). Measuring cell-generated forces: a guide to the available tools. Nature methods, 13(5), 415.

28. Roca-Cusachs, P., Conte, V., \& Trepat, X. (2017). Quantifying forces in cell biology. Nature cell biology, 19(7), 742-751.

29. Woo K., Fraser S. E. (1995) Order and coherence in the fate map of the zebrafish nervous system. Development 121, 2595-2609.

30. Loganathan, R., Little, C. D., Joshi, P., Filla, M. B., Cheuvront, T. J., Lansford, R., \& Rongish, B. J. (2014). Identification of emergent motion compartments in the amniote embryo. Organogenesis, 10(4), 350-364.

31. Chan, Chii J., Carl-Philipp Heisenberg, and Takashi Hiiragi (2017). Coordination of morphogenesis and cell-fate specification in development. Current Biology 27.18: R1024-R1035. 
32. Keller R. (2002) Shaping the vertebrate body plan by polarized embryonic cell movements. Science 298, 1950-1954.

33. Solnica-Krezel L., Sepich D. S. (2012) Gastrulation: making and shaping germ layers. Annual review of cell and developmental biology 28, 687-717.

34. Mitrossilis, D., Röper, J. C., Le Roy, D., Driquez, B., Michel, A., Ménager, C., ... \& Dempsey, N. M. (2017). Mechanotransductive cascade of Myo-II-dependent mesoderm and endoderm invaginations in embryo gastrulation. Nature communications, 8, 13883.

35. Amat F. et al. (2014) Fast, accurate reconstruction of cell lineages from large-scale fluorescence microscopy data. Nature methods 11,951-958.

36. Faure E. et al. (2016) A workflow to process 3D+time microscopy images of developing organisms and reconstruct their cell lineage. Nature Communications 78674.

37. Wagner, D. E., Weinreb, C., Collins, Z. M., Briggs, J. A., Megason, S. G., \& Klein, A. M. (2018). Single-cell mapping of gene expression landscapes and lineage in the zebrafish embryo. Science, 360(6392), 981-987.

38. Briggs, J. A., Weinreb, C., Wagner, D. E., Megason, S., Peshkin, L., Kirschner, M. W., \& Klein, A. M. (2018). The dynamics of gene expression in vertebrate embryogenesis at single-cell resolution. Science, 360(6392), eaar5780.

39. Zhang, J., Talbot, W. S., \& Schier, A. F. (1998). Positional cloning identifies zebrafish one-eyed pinhead as a permissive EGF-related ligand required during gastrulation. Cell, 92(2), 241-251.

40. Holzapfel, G. A. (2002) Nonlinear solid mechanics: a continuum approach for engineering science. Meccanica 37, 489-490. 
41. Chong M. S., Perry A. E., Cantwell B. J. (1990) A general classification of three-dimensional flow fields. Physics of Fluids A: Fluid Dynamics 2, 765-777.

42. Kimmel C. B. et al. (1995) Stages of embryonic development of the zebrafish. Developmental dynamics 203, 253-310.

43. England S. J., Blanchard G. B., Mahadevan L., Adams R. J. (2006) A dynamic fate map of the forebrain shows how vertebrate eyes form and explains two causes of cyclopia. Development, 133, 4613-4617.

44. Joore, J., Fasciana, C., Speksnijder, J. E., Kruijer, W., Destrée, O. H., van den Eijnden-van, A. J., ... \& Zivkovic, D. (1996). Regulation of the zebrafish goosecoid promoter by mesoderm inducing factors and Xwnt1. Mechanisms of development, 55(1), 3-18.

45. Delile J., Herrmann M., Peyriéras N., Doursat R. (2016) MecaGen: a cell-based computational model of embryogenesis coupling mechanical behavior and gene regulation. Nature Communications 7. In press.

46. He B., Doubrovinski K., Polyakov O., Wieschaus E. (2014) Apical constriction drives tissue-scale hydrodynamic flow to mediate cell elongation. Nature 508, 392-396.

47. Fleury V., Chevalier N. R., Furfaro F., Duband F. J. (2015) Buckling along boundaries of elastic contrast as a mechanism for early vertebrate morphogenesis. The European Physical Journal E $38,1-19$.

48. Moriyoshi K., Richards L. J., Akazawa C., O'Leary D. D., Nakanishi S. (1996) Labeling neural cells using adenoviral gene transfer of membrane-targeted GFP. Neuron 16, 255-260.

49. Shaner N. C., Steinbach P. A., Tsien R. Y. (2005) A guide to choosing fluorescent proteins. Nature methods 2, 905-909. 
50. Westerfield M. (2000) The zebrafish book: a guide for the laboratory use of zebrafish (Danio rerio) (University of Oregon Press)

51. So P. T., Dong C. Y., Masters B. R., Berland K. M. (2000) Two-photon excitation fluorescence microscopy. Annual review of biomedical engineering 2, 399-429

52. CRAMiR, H., \& Leadbetter, M. R. (1967). Stationary and Related Stochastic Processes. JohnWiley, New York.

53. Shadden, S. C., Lekien, F., \& Marsden, J. E. (2005). Definition and properties of Lagrangian coherent structures from finite-time Lyapunov exponents in two-dimensional aperiodic flows. Physica D: Nonlinear Phenomena, 212(3-4), 271-304.

54. Haller, G. (2015). Lagrangian coherent structures. Annual Review of Fluid Mechanics, 47, 137162.

55. Castro-González C. et al. (2014). A digital framework to build, visualize and analyze a gene expression atlas with cellular resolution in zebrafish early embryogenesis. PLoS Computational Biology, 10, e1003670. 


\section{Figure 1: Construction of the biomechanical map from the reconstructed cell lineage}

(A, B, C) WT specimen wtl imaged live between 6 and 12 hpf, animal pole (AP) view, 3D rendering with the Mov-IT software. Scale bar $50 \mu m$. (A) 3D+time microscopy imaging of zebrafish embryos, nuclear staining (green), snapshots at 8, 10 and 12 hpf indicated top right. Insets schematize the imaged volume and the xy field of view. (B) Regularized displacement field (see Methods, Supplementary Fig. 2). Colormap for the speed intensity from 0 (dark blue) to 2 (white) $\mu \mathrm{m} / \mathrm{sec}$. (C) Left Panel: 3D rendering of the original cell tracking; right panel: trajectories completed using the regularized velocity field. Each cell nucleus approximate center is represented by a cube, the cell trajectory for the next 5 time steps is represented by a line. (D) Schematics of a typical cell distribution in 2D (top-left panel); deformation patterns quantified with the IDG tensor, instantaneous descriptors indicated top right of each panel: expansion $(P>0)$, compression $(P<$ 0 ), simple shear (bottom left panel) and pure shear (bottom middle panel) $\left(Q_{d}\right)$, rotation $(D>0)$. (E) Schematic representation of the computation of instantaneous and cumulative descriptors from the IDG tensor field. Eulerian compression descriptor P(Trajs) at each time step along the cell trajectory. Lagrangian compression descriptor $\Delta V_{t_{i n i}}(\operatorname{Trajs})$ is calculated from an initial time point $t_{\text {ini }}$. Colormap: from compression (red) to expansion (blue). (F) Schematic representation of instantaneous (left panel) and cumulative (right panel) Lagrangian Biomechanical Profiles (LPBs) for a selection of cells and their trajectories between $8\left(t_{\text {ini }}\right)$ to $14 \mathrm{hpf}$. Colormap: from compression (red) to expansion (blue). (G) Overview of the two-step clustering strategy that leads to the identification of Langrangian biomechanical domains. First, Lagrangian Biomechanical Profiles (LPBs) displayed in (F) are split into 3 main clusters called CLBPs (dark grey, brown, light grey). Second, CLBPs are used to classify trajectories and label cells accordingly (red, magenta, green and yellow). 


\section{Figure 2: wt and zoep ${ }^{t z 57 / t z 57}$ biomechanical landmarks of gastrulation}

Comparison between a wild-type embryo wt 1 (columns 1 and 3 ) and a zoep ${ }^{t z 57 / t z 57}$ mutant embryo oepl (columns 2 and 4). Time point (7, 8, 9, 10, 11 and 12 hpf) indicated to right. Descriptor indicated top left of each pair (wtl and oepl) of panels. Velocity field $\left(v_{T R}\right)$ colormap from from dark (zero speed) to white $(2 \mu \mathrm{m} / \mathrm{sec}) . P$ colormap goes from compression (red) to neutral (grey) to expansion (blue). $Q_{d}$ colormap from dark (no distortion) to bright (maximum distortion). Comparison at $9 \mathrm{hpf}$ (top-left panel) features velocity field (up) and $P$ (down). Rotation discriminant $D$ colormap from blue (no rotation) to green-yellow (maximum rotation observed). Scale bar $50 \mu m$.

\section{Figure 3: Cumulative descriptors of $w t$ development}

Comparison between Eulerian and Lagrangian descriptors for wild-type embryo wt1. Time points $(9,10,11$ and $12 \mathrm{hpf})$ indicated top right of each panel. 6 sub-panels per time point with descriptor indicated top left. Similar display in the 4 panels with Eulerian descriptors $\left(P Q_{d},, D\right)$ left column in each subpanel and Lagrangian descriptors $\left(\Delta V, \Delta \gamma_{1}\right.$ and $\left.\Delta \alpha\right)$ on the right. Eulerian descriptors, same colormap as in Figure 2: $P$ colormap from compression (red) to neutral (grey) to expansion (blue); $Q_{d}$ from dark (no distortion) to bright (maximum distortion); $D$ colormap goes from blue (no rotation) to green-yellow (maximum rotation). Lagrangian descriptors: $\Delta V$ colormap from compression (red) to neutral (grey) to expansion (blue); $\Delta \gamma_{1}$ colormap from dark (no distortion) to bright (maximum distortion); $\Delta \alpha$ colormap from blue (no rotation) to green-yellow (maximum rotation). Scale bar $50 \mu m$. 
Figure 4: Quantitative comparison of Lagrangian Biomechanical Profiles (LBPs) in a cohort of zebrafish embryos

(A, B) Comparison of the LBPs' mean (line) and variance (shaded area), calculated for selected cell populations (selection at the tail bud stage, Supplementary Fig. 8, Supplementary Movie 22), hypoblast (red) and epiblast (blue) in embryos $w t 1-w t 5$ (row 1 to 5 respectively). (A) Instantaneous LBPs. Time in hpf. The vertical dashed black line indicates the onset of epiblast compression chosen as the initial time $\left(t_{\text {ini }}\right)$. (B) LBPs cumulated from $\left(t_{\text {ini }}\right)$ for the next 6 hours, mean (line) and variance (shaded area). The 5 plots are aligned in time at $t_{i n i}$. Hypoblast was not analyzed in embryo $w t 2$ as it was not present at $t_{i n i}$ because of the embryo position in the field of view.

Figure 5: Order and coherence of the Lagrangian biomechanical map in normal and oep mutant embryos

(A) Mean (m) for each of the three LBP clusters (number of clusters identified as optimal, Supplementary Fig. 10-12) defined the CLBPs for each Lagrangian descriptor (e.g. $\Delta V_{m 1}$ to $\Delta V_{m 3 \text {, }}$ etc) for the wild type embryo (top row) and the mutant (bottom row); arbitrary color code to distinguish the different CLBPs. For $\Delta V$, values higher than 1 mean expansion and bellow 1 compression. For $\Delta \gamma_{1}$ and $\Delta \gamma_{2}$, the value range goes from no distortion (0) to maximum of distortion (3). For $\Delta \alpha$, the value represents the angle of rotation between $0^{\circ}$ and $360^{\circ}$. (B) Snapshots of the Lagangian biomechanical map built for $w t 1$ and oep 1 embryos, cells at $t_{\text {ini }}=8$ hpf were selected in Mov-IT and labeled according to their Lagrangian biomechanical signature; the corresponding color code was propagated along the cell lineage; different time points indicated top right. At 8 and $12 \mathrm{hpf}$, unlabeled nuclei in blue. At 10 and $13 \mathrm{hpf}$, labeled nuclear centers are displayed together with the membrane raw data (3D rendering in blue), upper sections were removed down to $65 \mu \mathrm{m}$ below the embryo surface. Scale bar $50 \mu \mathrm{m}$. First row: wt 1 embryo, four clusters 
identified at $t_{\text {ini }}=8 \mathrm{hpf}$ (Supplementary Movie 23-26). Second row: oep1 embryo, three clusters identified at $t_{i n i}=8 \mathrm{hpf}$.

\section{Figure 6: The gsc gene expression domain matches a biomechanical field}

wt6 embryo from the transgenic line gsc:egfp (Methods) imaged from 8 to $13 \mathrm{hpf.} \mathrm{(A)}$ Quantification of gsc reporter expression at the single cell level (Methods, Supplementary Table 1) and clustering of cell populations according to the reporter expression level using $k$-means clustering ( $k=3$ green $-g s c_{m 1^{-}}$, light blue $-g s c_{m 2^{-}}$and magenta $-g s c_{m 3^{-}}$- for the mean expression level in each cluster). (B) Average value of nuclear staining intensity along cell trajectories for each of the clusters identified in (A): $n u c_{m 1}, n u c_{m 2}$ and $n u c_{m 3}$. (C) Average value of the $g s c$ reporter expression after being corrected by the nuclear staining intensity $\left(\operatorname{gscN}_{m 1}, g s c N_{m 2}\right.$ and $\left.g s c N_{m 3}\right)$. (D, E) Visualization of the Lagrangian Biomechanical Maps with the Mov-IT software. Cells at $8 \mathrm{hpf}$ are labelled according to their cluster and the color propagated along the cell lineage. Scale bar $50 \mu m$. (D) Spatial organization of the three gsc expression clusters (green $-g s c_{m 1^{-}}$, light blue $-g s c_{m 2^{-}}$and magenta $\left.-g s c_{m 3}\right)$. Snapshots at 8 and $10 \mathrm{hpf}$ indicated top right. (E) Lagrangian Biomechanical Map for wt6 computed and displayed as for wt 1 and oep 1 in Figure 5B $\left(t_{i n i}=8 \mathrm{hpf}\right)$. Snapshots at $8 \mathrm{hpf}$ and $10 \mathrm{hpf}$ indicated top right. 


\section{Figures}

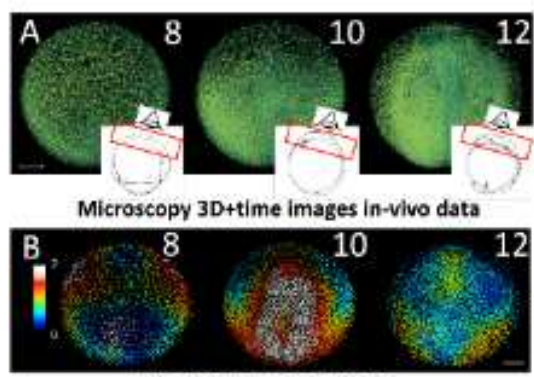

Regularized velocity field
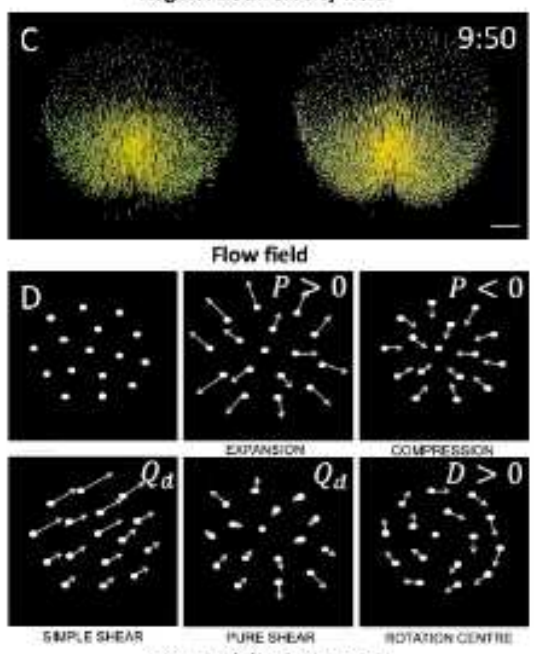

Tensor of displacements

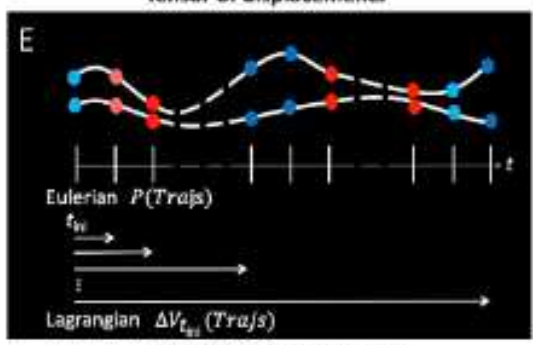

Lagrangian representation

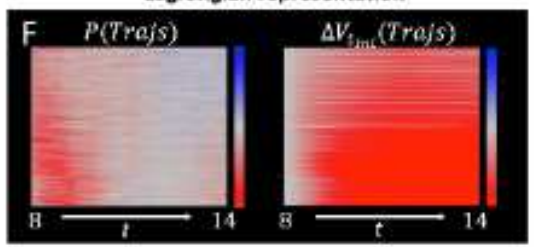

Lagrangian Biomechanical Profiles

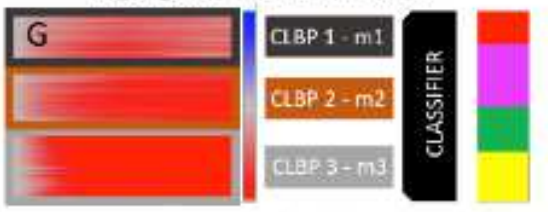

Categorization LBP

\section{Figure 1}

Construction of the biomechanical map from the reconstructed cell lineage (A, B, C) WT specimen wt1 imaged live between 6 and $12 \mathrm{hpf}$, animal pole (AP) view, 3D rendering with the Mov-IT software. Scale bar $50 \mu \mathrm{m}$. (A) 3D+time microscopy imaging of zebrafish embryos, nuclear staining (green), snapshots at 
8,10 and $12 \mathrm{hpf}$ indicated top right. Insets schematize the imaged volume and the xy field of view. (B) Regularized displacement field (see Methods, Supplementary Fig. 2). Colormap for the speed intensity from 0 (dark blue) to 2 (white) $\mu \mathrm{m} / \mathrm{sec}$. (C) Left Panel: 3D rendering of the original cell tracking; right panel: trajectories completed using the regularized velocity field. Each cell nucleus approximate center is represented by a cube, the cell trajectory for the next 5 time steps is represented by a line. (D) Schematics of a typical cell distribution in 2D (top-left panel); deformation patterns quantified with the IDG tensor, instantaneous descriptors indicated top right of each panel: expansion $(P>0)$, compression $(P<0)$, simple shear (bottom left panel) and pure shear (bottom middle panel) $(Q d)$, rotation $(D>0)$. $(E)$ Schematic representation of the computation of instantaneous and cumulative descriptors from the IDG tensor field. Eulerian compression descriptor P(Trajs) at each time step along the cell trajectory. Lagrangian compression descriptor $\Delta$ Vtini (Trajs) is calculated from an initial time point tini. Colormap: from compression (red) to expansion (blue). (F) Schematic representation of instantaneous (left panel) and cumulative (right panel) Lagrangian Biomechanical Profiles (LPBs) for a selection of cells and their trajectories between 8 (tini) to $14 \mathrm{hpf}$. Colormap: from compression (red) to expansion (blue). (G) Overview of the two-step clustering strategy that leads to the identification of Langrangian biomechanical domains. First, Lagrangian Biomechanical Profiles (LPBs) displayed in (F) are split into 3 main clusters called CLBPs (dark grey, brown, light grey). Second, CLBPs are used to classify trajectories and label cells accordingly (red, magenta, green and yellow). $\mu \mathrm{m}$ 

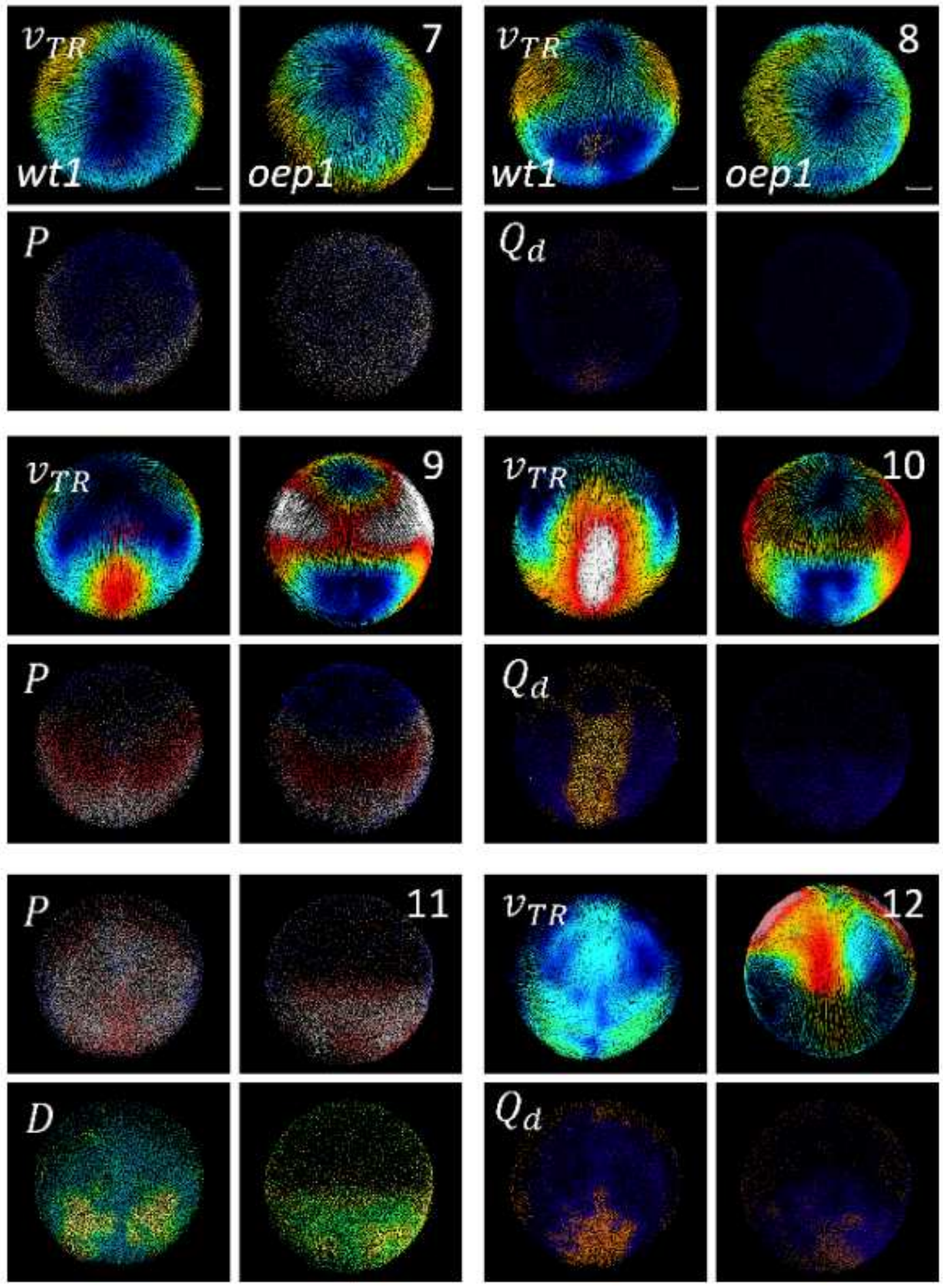

Figure 2

wt and zoeptz57/tz57 biomechanical landmarks of gastrulation Comparison between a wild-type embryo wt1 (columns 1 and 3 ) and a zoeptz57/tz57 mutant embryo oep1 (columns 2 and 4). Time point (7, 8, 9, 10, 11 and $12 \mathrm{hpf}$ ) indicated to right. Descriptor indicated top left of each pair (wt1 and oep1) of panels. Velocity field (vTR) colormap from from dark (zero speed) to white $(2 \mu \mathrm{m} / \mathrm{sec})$. P colormap goes from compression (red) to neutral (grey) to expansion (blue). Qd colormap from dark (no distortion) to bright 
(maximum distortion). Comparison at $9 \mathrm{hpf}$ (top-left panel) features velocity field (up) and P (down). Rotation discriminant $\mathrm{D}$ colormap from blue (no rotation) to green-yellow (maximum rotation observed). Scale bar $50 \mu \mathrm{m}$.
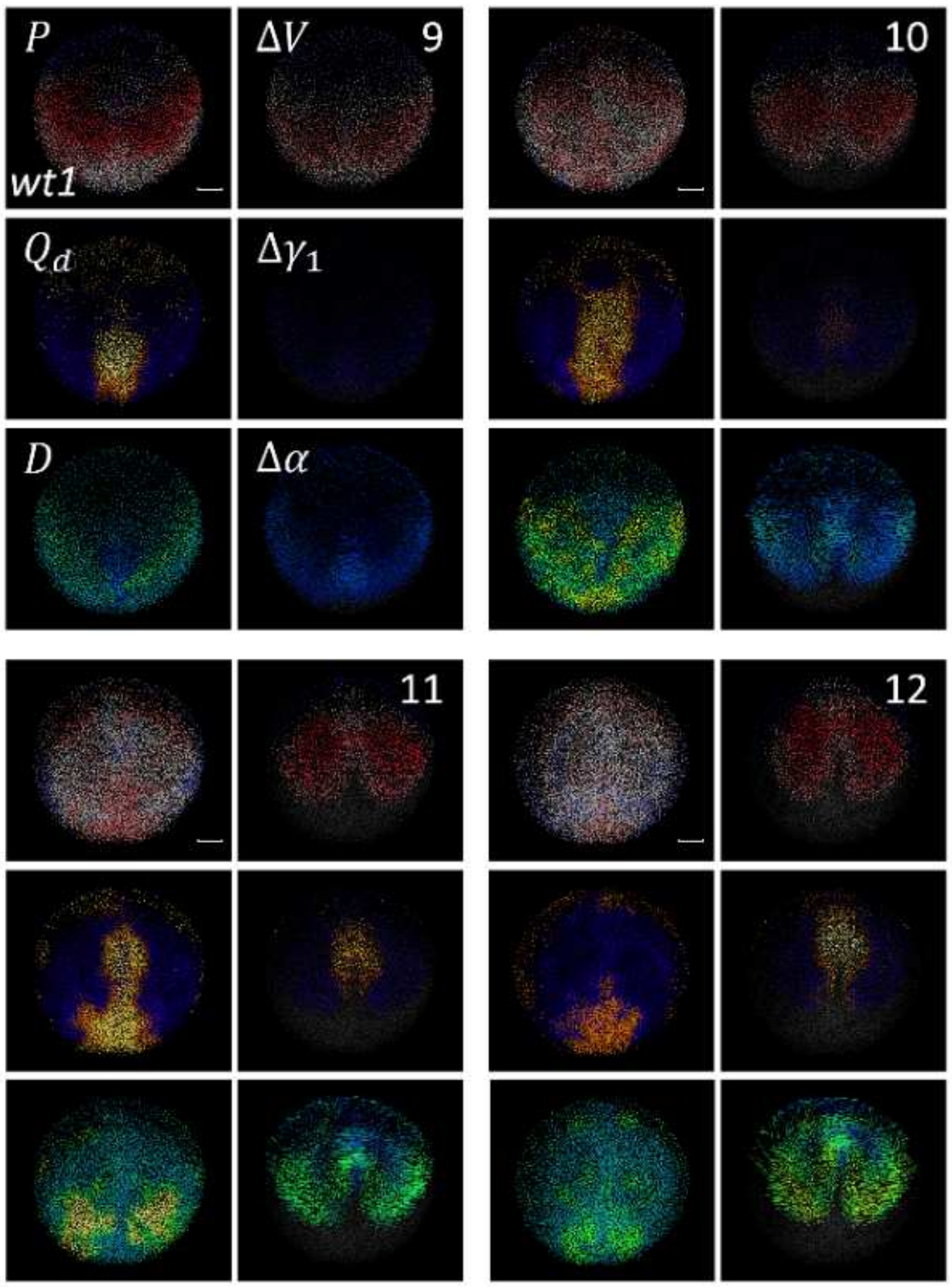

Figure 3

Cumulative descriptors of wt development Comparison between Eulerian and Lagrangian descriptors for wild-type embryo wt1. Time points $(9,10,11$ and $12 \mathrm{hpf})$ indicated top right of each panel. 6 sub-panels 
per time point with descriptor indicated top left. Similar display in the 4 panels with Eulerian descriptors (PQd, , D) left column in each subpanel and Lagrangian descriptors $(\Delta V, \Delta Y 1$ and $\Delta \mathrm{a})$ on the right. Eulerian descriptors, same colormap as in Figure 2: P colormap from compression (red) to neutral (grey) to expansion (blue); Qd from dark (no distortion) to bright (maximum distortion); D colormap goes from blue (no rotation) to green-yellow (maximum rotation). Lagrangian descriptors: $\Delta V$ colormap from compression (red) to neutral (grey) to expansion (blue); $\Delta y 1$ colormap from dark (no distortion) to bright (maximum distortion); $\Delta \mathrm{a}$ colormap from blue (no rotation) to green-yellow (maximum rotation). Scale bar $50 \mu \mathrm{m}$.

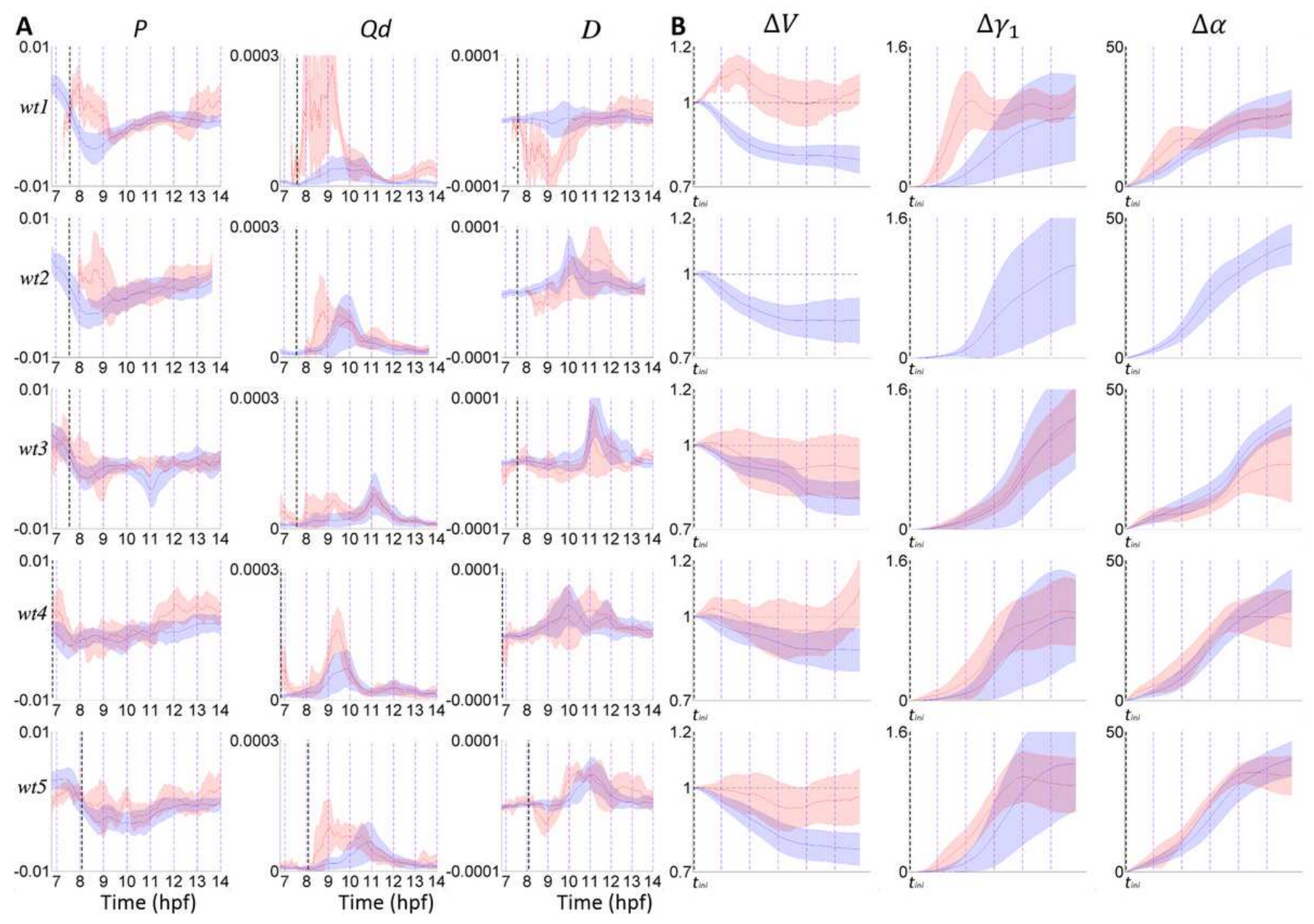

Figure 4

Quantitative comparison of Lagrangian Biomechanical Profiles (LBPs) in a cohort of zebrafish embryos (A, B) Comparison of the LBPs' mean (line) and variance (shaded area), calculated for selected cell populations (selection at the tail bud stage, Supplementary Fig. 8, Supplementary Movie 22), hypoblast (red) and epiblast (blue) in embryos wt1-wt5 (row 1 to 5 respectively). (A) Instantaneous LBPs. Time in hpf. The vertical dashed black line indicates the onset of epiblast compression chosen as the initial time (tini). (B) LBPs cumulated from (tini) for the next 6 hours, mean (line) and variance (shaded area). The 5 
plots are aligned in time at tini. Hypoblast was not analyzed in embryo wt2 as it was not present at tini because of the embryo position in the field of view.

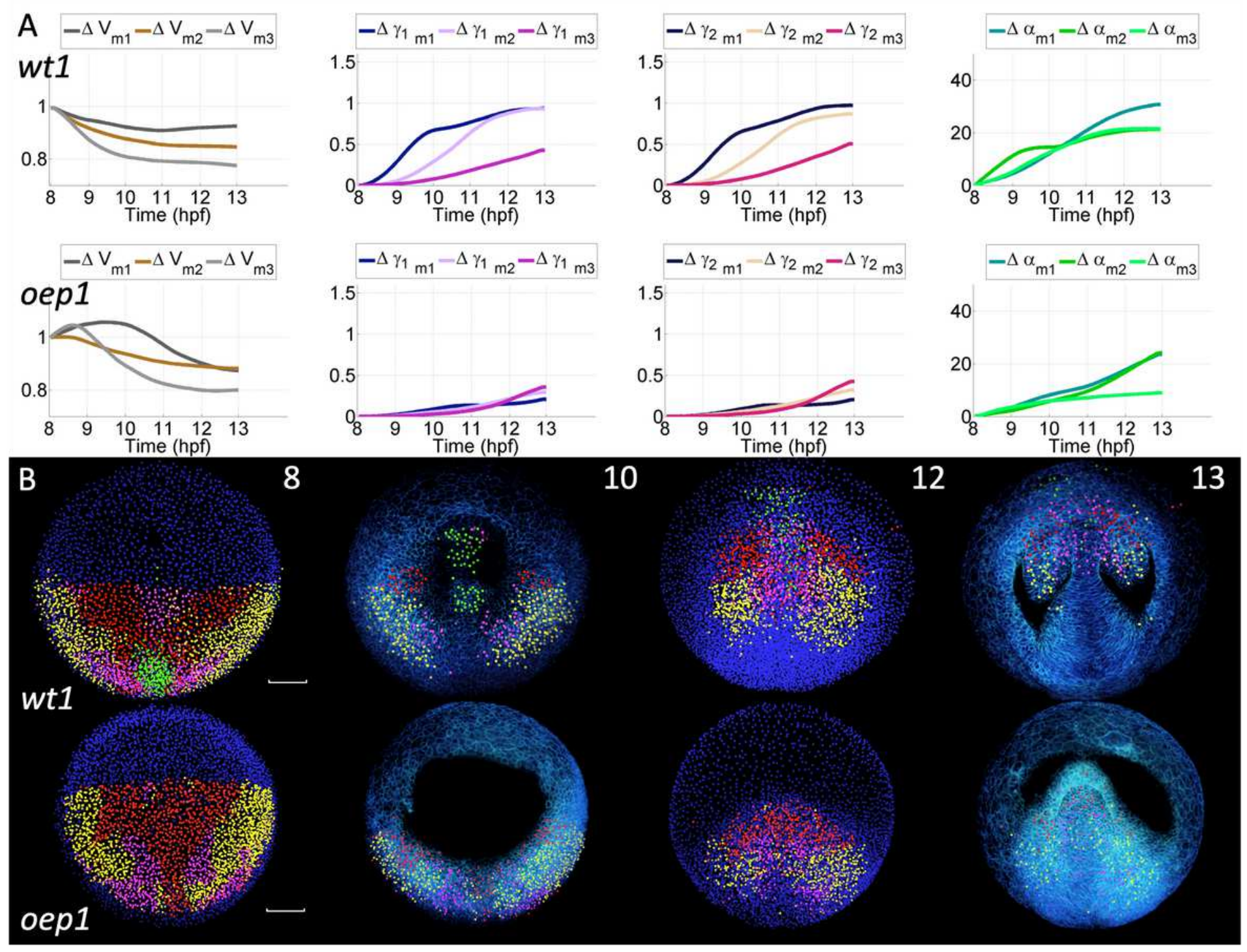

\section{Figure 5}

Order and coherence of the Lagrangian biomechanical map in normal and oep mutant embryos (A) Mean (m) for each of the three LBP clusters (number of clusters identified as optimal, Supplementary Fig. 1012) defined the CLBPs for each Lagrangian descriptor (e.g. $\Delta \mathrm{Vm} 1$ to $\Delta \mathrm{Vm} 3$, etc) for the wild type embryo (top row) and the mutant (bottom row); arbitrary color code to distinguish the different CLBPs. For $\Delta \mathrm{V}$, values higher than 1 mean expansion and bellow 1 compression. For $\Delta y 1$ and $\Delta y 2$, the value range goes from no distortion (0) to maximum of distortion (3). For $\Delta a$, the value represents the angle of rotation between $0^{\circ}$ and $360^{\circ}$. (B) Snapshots of the Lagangian biomechanical map built for wt1 and oep1 embryos, cells at tini $=8 \mathrm{hpf}$ were selected in Mov-IT and labeled according to their Lagrangian biomechanical signature; the corresponding color code was propagated along the cell lineage; different time points indicated top right. At 8 and 12 hpf, unlabeled nuclei in blue. At 10 and 13 hpf, labeled nuclear centers are displayed together with the membrane raw data (3D rendering in blue), upper sections were 
removed down to $65 \mu \mathrm{m}$ below the embryo surface. Scale bar $50 \mu \mathrm{m}$. First row: wt1 embryo, four clusters identified at tini = $8 \mathrm{hpf}$ (Supplementary Movie 23-26). Second row: oep1 embryo, three clusters identified at tini $=8 \mathrm{hpf}$.
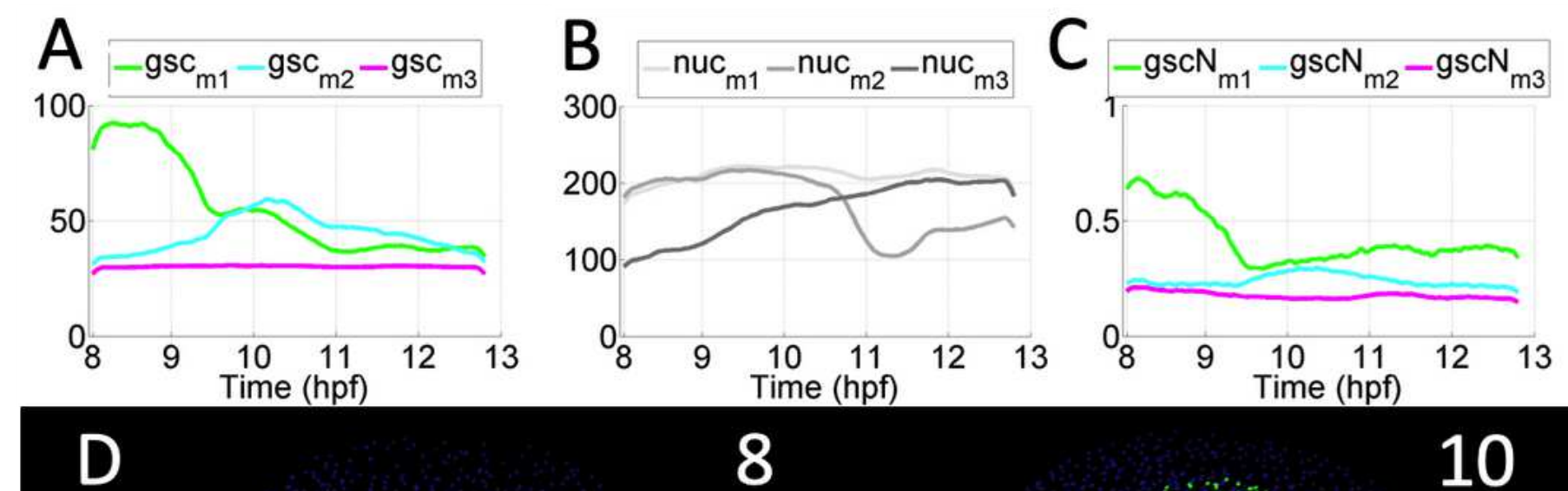

\section{8}
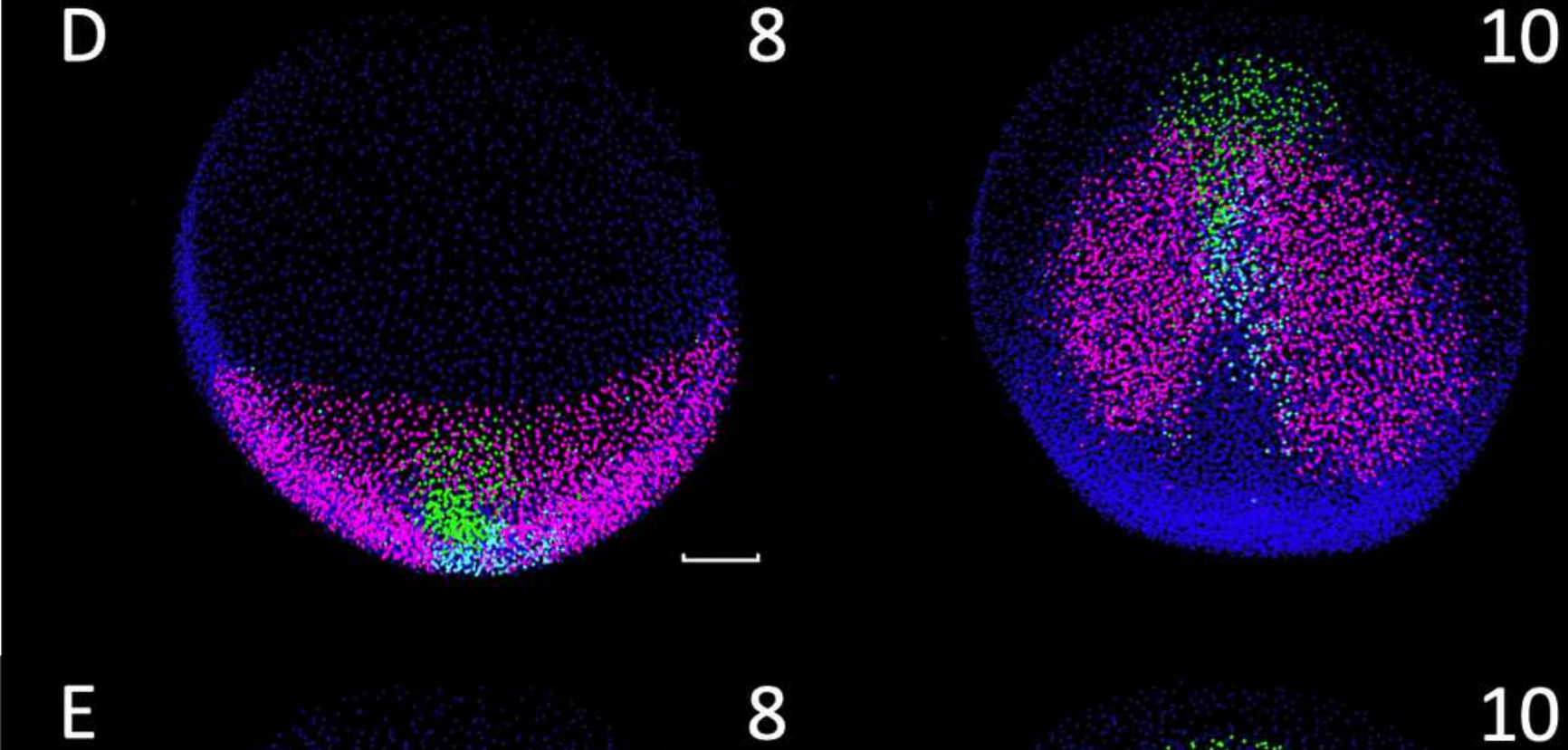

E $\quad 8$

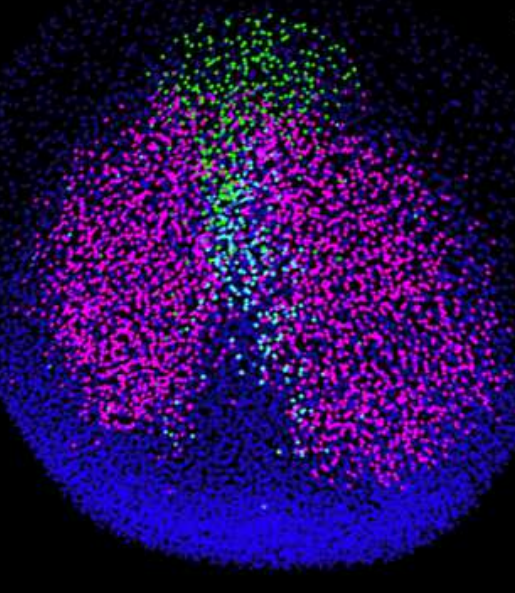

10

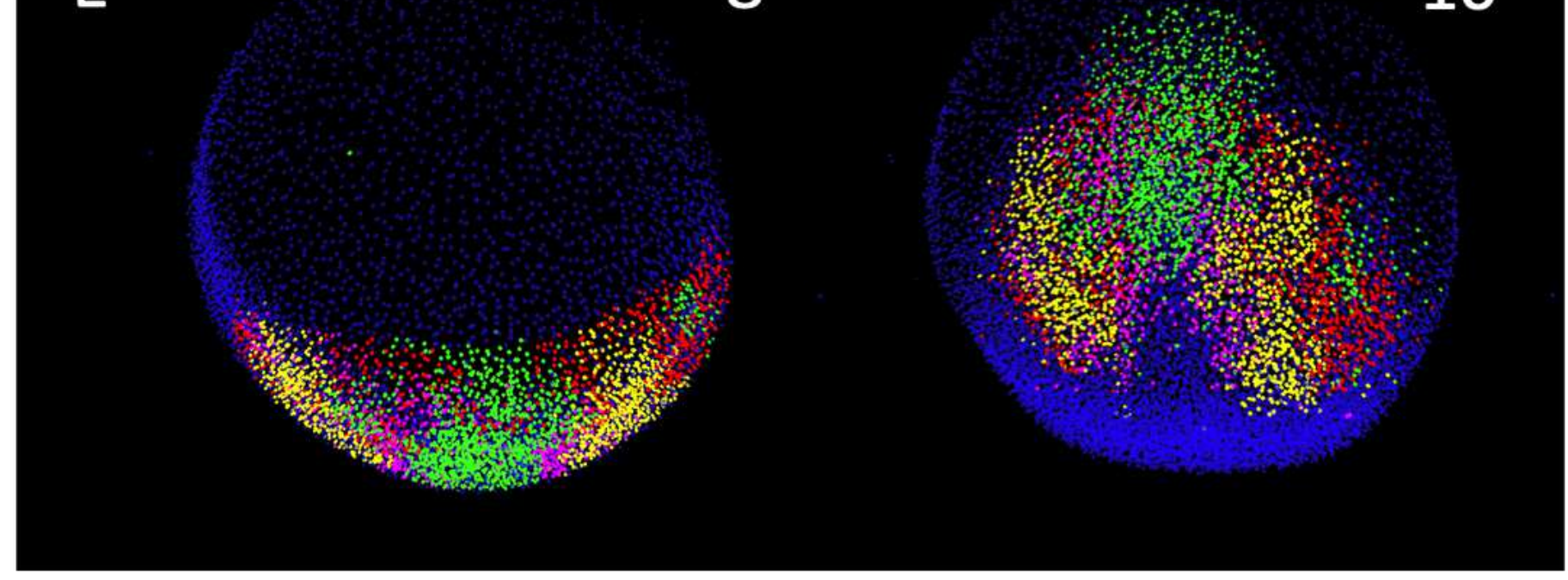

Figure 6

The gsc gene expression domain matches a biomechanical field wt6 embryo from the transgenic line gsc:egfp (Methods) imaged from 8 to $13 \mathrm{hpf}$. (A) Quantification of gsc reporter expression at the single 
cell level (Methods, Supplementary Table 1) and clustering of cell populations according to the reporter expression level using k-means clustering ( $k=3$ green -gscm1-, light blue -gscm2- and magenta -gscm3for the mean expression level in each cluster). (B) Average value of nuclear staining intensity along cell trajectories for each of the clusters identified in (A): nucm1, nucm2 and nucm3. (C) Average value of the gsc reporter expression after being corrected by the nuclear staining intensity (gscNm1, gscNm2 and gscNm3). (D, E) Visualization of the Lagrangian Biomechanical Maps with the Mov-IT software. Cells at 8 $\mathrm{hpf}$ are labelled according to their cluster and the color propagated along the cell lineage. Scale bar 50 $\mu \mathrm{m}$. (D) Spatial organization of the three gsc expression clusters (green -gscm1-, light blue -gscm2- and magenta -gscm3). Snapshots at 8 and $10 \mathrm{hpf}$ indicated top right. (E) Lagrangian Biomechanical Map for wt6 computed and displayed as for wt1 and oep 1 in Figure 5B (tini $=8 \mathrm{hpf}$ ). Snapshots at $8 \mathrm{hpf}$ and 10 hpf indicated top right

\section{Supplementary Files}

This is a list of supplementary files associated with this preprint. Click to download.

- PastorEscuredoetalSuplnformationNatComms.docx 